\title{
Associations between low muscle mass, blood-borne nutritional status and mental health in older patients
}

\author{
Salah Gariballa ${ }^{1,2^{*}}$ and Awad Alessa
}

\begin{abstract}
Background: Although low muscle mass is an important predictor of increased physical morbidity in older patients, information on its impact on mental health and well-being is lacking. The first aim of this report is to look for associations if any between low muscle mass and mental health of older people in clinical practice. The second aim is to study underlying mechanisms including nutritional status.

Methods: In this prospective longitudinal study we randomly selected and studied 432 hospitalized older patients' baseline demographic data, clinical characteristics and nutritional status on admission, at 6 weeks and at 6 months. Low muscle mass was diagnosed using anthropometric measures based on the European Working Group criteria. Mental health outcome measures including cognitive state, depression symptoms and quality of life were also measured.
\end{abstract}

Results: Out of 432 patients assessed 44 (10\%) were diagnosed with low muscle mass. Patients diagnosed with low muscle mass at admission and over a 6-month follow up period had significantly poor cognitive function, quality of life and increased depression symptoms compared with those with normal muscle mass. After adjustment for poor prognostic indicators, age, disability, severity of acute illness and low muscle mass were associated with poor cognitive function and quality of life and higher depression symptoms in older patients over a 6 months period $(p<0.05)$. Although patients with low muscle mass had lower micronutrient concentrations compared to those patients with normal muscle mass, only serum albumin showed significant correlations with quality of life at admission and depression symptoms at 6 weeks.

Conclusion: Low muscle mass is associated with poor blood-borne poor nutritional status and mental health in hospitalized older patients, however, this is partly explained by underlying co morbidity.

Keywords: Muscle mass, Mental health, Older people, Acute illness

\section{Background}

Despite growing evidence that low muscle mass is common and associated with increasing morbidity and mortality in older people, there is a lack of good-quality data of the effect of low muscle mass diagnosis on mental health and quality of life measures and their response to treatment [1-3]. Although a relationship between changes in body composition including muscle mass and mental well-being in older people has been postulated

\footnotetext{
* Correspondence: s.gariballa@uaeu.ac.ae

${ }^{1}$ Internal Medicine, Faculty of Medicine \& Health Sciences, United Arab Emirates University (UAEU), Al Ain, United Arab Emirates

${ }^{2}$ University of Sheffield, Sheffield, UK
}

before, however supporting research evidence is weak [4]. Skeletal muscle cells are known to be metabolically active and secrete a number of substances that communicate directly with a number of organs including the brain and may therefore be responsible for the beneficial effects of muscle contraction/exercise on mental health [5]. Specifically postulated common underlying pathophysiological mechanisms linking changes in muscle mass with poor mental health and well-being include inflammation, oxidative stress, malnutrition and physical inactivity all are common and associated with ill health in older people [6]. A recent cross-sectional study on community -living older people has revealed that 
subjects with lower appendicular skeletal muscle mass had lower cognitive functioning scores than did participants with higher muscle mass scores [7]. We have recently reported that poor muscle strength in hospitalized older people is associated with poor physical and mental health [8]. A recent study however reported that poor muscle mass but not poor strength is associated with malnutrition in hospitalized older patients at admission [7]. Given the known link between malnutrition and poor mental health, the aim of this report is to present data on the relationship between low muscle mass, mental health and quality of life and underlying blood-borne nutritional status in hospitalized older patients.

\section{Methods}

Subjects included in this research report were acutely illhospitalized older patients with common diagnoses who were part of for a randomized controlled trial [9] All subjects were admitted to Barnsley District General Hospital, UK. Barnsley District General Hospital serves a total population of 234,000 . It has 650 beds and the medical unit has 250 beds for acute medical admissions. Patients were first, identified through the computerised hospital admission databases of all patients. When first admitted all patients have an individualised computerised plan created. The medical notes of those identified from the database were examined and eligible patients approached. Patients aged 65 years or older admitted with common diagnoses of ischaemic heart disease, heart failure, stroke, chest, urinary tract and blood infection, chronic obstructive lung disease, falls, syncope, and fractured limbs were included. Patients had to sign informed consent form to participate in the index study. Patient excluded from the study were those with severe medical or psychiatric illness including those living in institution. The study design was approved by Barnsley (UK) research ethics committee (REF: 04/Q2304/50). Data collected from medical records included demographic and clinical data admission diagnosis, previous illnesses, disability, medications, smoking and alcohol intake. All patients had three assessments the first within $72 \mathrm{~h}$ of admission in hospital and at 6 weeks and at 6 months either in hospital or in the community. Disability was assessed using the Barthel which scores 10 functions on a scale 0 (fully dependent) to 20 (independent). Standard anthropometric, hematological and biochemical data were used to assess nutritional status [9]. Method for determination of specific micronutrients concentrations has been published before [10]. Low muscle mass was diagnosed using The European Working Group on Sarcopenia in Older people (EWGSOP) criteria $[2,3]$. The muscle mass was measured by midarm muscle circumference (MAMC) using the following formula: MAMC $=$ MAC - $\left(\begin{array}{lll}3.14 & \mathrm{x} & \text { triceps skinfold }\end{array}\right.$ thickness). Low muscle mass was classified as MAMC less $21.1 \mathrm{~cm}$ and $19.2 \mathrm{~cm}$ in men and women respectively. Cognitive state was assessed by the abbreviated mental test questionnaire (AMT) [11]. The 15 item Geriatric Depression questionnaire (GDS) was used to measure depression symptoms [11]. The validated Medical Outcomes Study 36-items (SF-36) General Health Survey questionnaire used to assess quality of life [12].

\section{Statistical analysis}

The independent student-t test or the nonparametric Mann-u-Whitney (SPSS version 24) were used to test differences in nutritional micronutrient variables between patients with low muscle mass and those patients with normal muscle mass. A $p$-value of $<0.05$ is regarded as statistically significant. A forward stepwise multiple regression analysis was performed to determine the influence blood-borne nutritional markers on mental health outcome measures including cognitive state, depression symptoms and quality of life at admission and at 6 weeks. We also used multivariate analysis to determine the influence of low muscle mass on mental health and quality of life after adjusting for a number of independent poor prognostic clinical indicators including age, gender, disability, comorbidity (previous illnesses), and severity of acute illness (inflammation) measured using CRP.

\section{Results}

All 432 acutely ill older patients aged 65 to 92 years [211 (49\%) female] admitted to hospital and followed up for period of 6 months were included in this analysis. Among the 432 patients recruited 42 (10\%) had low muscle mass at baseline. Corresponding figures at 6 weeks and at 6 months were $15(10 \%)$ and 9 (6\%). Exclusions were due to early discharge, death or inability to provide outcome data at follow up visits. Patients with low muscle mass at admission and at 6 weeks and 6 months follow up had significantly poor cognitive function, quality of life and/or increased depression symptoms compared with patients with normal muscle mass (Figs. 1, 2 and 3). Multiple regression analysis for the association between low muscle mass and other clinical indicators at admission revealed significant correlations between age, disability, low muscle mass and cognitive function $(p<0.05)$. Although tissue inflammation showed significant associations with both cognitive function and depression symptoms, chronic illness revealed significant and independent associations with cognitive function, depression symptoms and quality of life (Table 1). Table 2 shows nutritional biomarkers in patients with low muscle mass compared to those patients with normal muscle mass. The results show lower concentrations of nutritional biomarkers in patients with 


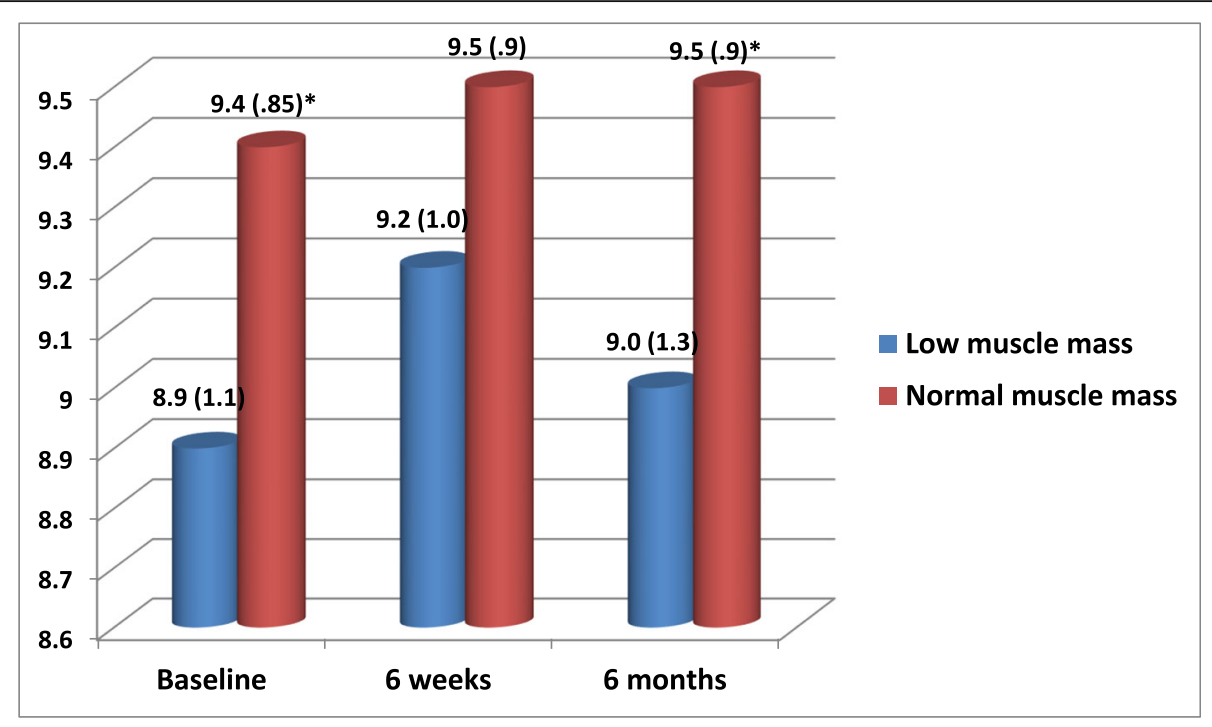

$* \mathrm{P}(2$ tailed $)<0.05$

AMT $=$ Abbreviated mental test (maximum score is 10. A score of 6 or less indicates cognitive deficit)

Fig. 1 Cognitive state during both acute illness and recovery for study patients diagnosed with Low muscle mass compared with those normal muscle mass

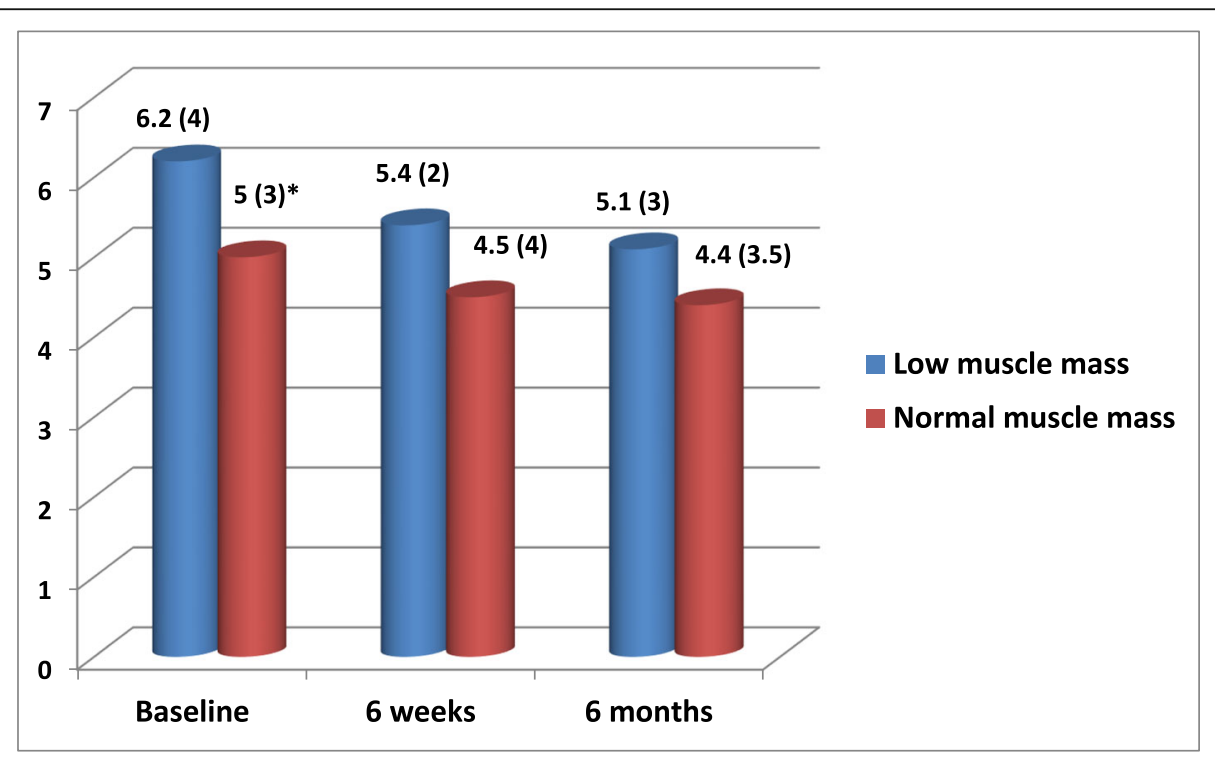

*P $(2$ tailed $)<0.05$

GDS $=$ Geriatric Depression score $(0-4=$ no depression; 5-10 = mild depression; $\geq 11=$ severe depression)

Fig. 2 Depression symptoms during both acute illness and recovery for study patients diagnosed with low muscle mass compared with those with normal muscle mass 


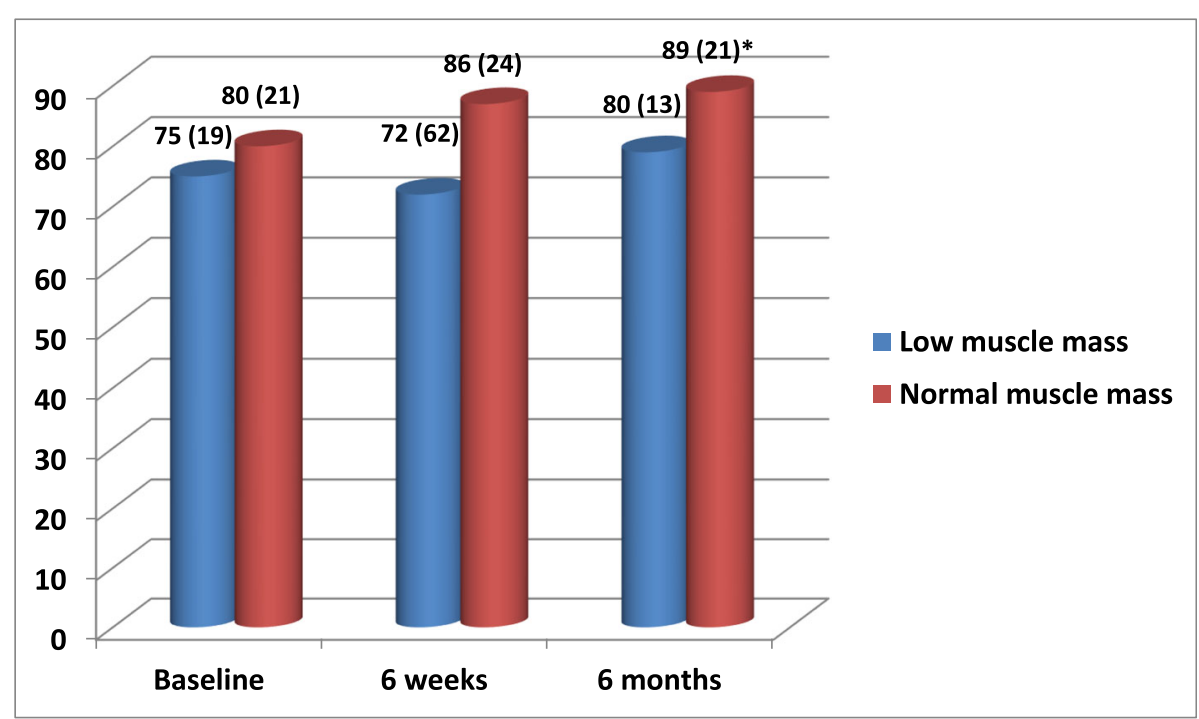

* $\mathrm{P}(2$ tailed $)<0.05$

SF-36=Quality of life General Health Survey (measured on a 0 to $100+($ good health)

Fig. 3 Quality of life during both acute illness and recovery for study patients diagnosed with Low muscle mass compared with those with normal muscle mass

low muscle mass but the differences were only statistically significant for baseline serum albumin, plasma zinc and red cell folate only $(p<0.05)$. Ascorbic acid, zinc, lycopene, folate, and retinol were also significantly lower in patients with low muscle mass at 6 weeks (Table 2). We found no statistically significant associations between nutritional biomarker concentrations and mental health or quality of life scores except for serum albumin on quality of life at admission and depression symptoms at 6 weeks (Tables 3 and 4).

\section{Discussion}

In this analysis, we found that low muscle mass was associated with poor mental health. However age, disability, chronic illness and tissue inflammation a marker of the severity of acute illness were also independently associated with poor cognitive function, increased depression symptoms and/or poor quality of life. We also found that patients with low muscle mass had poor nutritional biomarkers including B-group vitamins, antioxidants and trace elements.

Although poor muscle mass is known to be independently associated with increasing age and comorbidity and consequently leads to increased disability, morbidity and mortality information, on its effect on mental health and well-being is lacking [2, 3, 13]. Furthermore the contribution of poor nutritional biomarkers including Bgroup vitamins, antioxidants and trace elements in

Table 1 Multiple regression result of age, gender, disability, co-morbidity, severity of acute illness and presence of low muscle mass on AMT, GDS and SF-36 on admission

\begin{tabular}{|c|c|c|c|c|c|c|}
\hline & \multirow{2}{*}{$\begin{array}{l}\text { Abbreviated mental test } \\
\text { score (AMT) } \\
\text { Standardized } \\
\text { Regression coefficient } \\
(95 \% \text { C.I) }\end{array}$} & \multirow[b]{2}{*}{$P$ value } & \multirow{2}{*}{$\begin{array}{l}\text { Geriatric Depression Score (GDS) } \\
\text { Standardized Regression } \\
\text { coefficient ( } 95 \% \text { C.I) }\end{array}$} & \multirow[b]{2}{*}{$P$ value } & \multirow{2}{*}{$\begin{array}{l}\text { Quality of life Scores (SF-36) } \\
\text { Standardized Regression } \\
\text { coefficient ( } 95 \% \text { C.I) }\end{array}$} & \multirow[b]{2}{*}{$P$ value } \\
\hline & & & & & & \\
\hline Age (years) & $-.12(-.03$ to -.003$)$ & $0.021^{*}$ & -.01 (-.06 to .055) & 0.880 & $.001(-.36$ to .37$)$ & 0.987 \\
\hline Gender & $-.07(-.30$ to .05$)$ & 0.158 & .001 (-.73 to .73) & 0.992 & $-.027(-5.6$ to 3.3$)$ & 0.619 \\
\hline Barthel score & $.12(-.003$ to .043$)$ & $0.025^{*}$ & $-.15(-.20$ to -.033$)$ & $0.006^{*}$ & $.12(.03$ to 1.04$)$ & $0.039^{*}$ \\
\hline Chronic illnesses & $.002(-.06$ to .06$)$ & 0.962 & $.06(-.11$ to .40$)$ & 0.255 & $-.16(-3.7$ to -.74$)$ & $0.004^{*}$ \\
\hline CRP $(\mathrm{mg} / \mathrm{L})+$ & .11 (.00 to .003$)$ & $0.033^{*}$ & $-.18(-.014$ to -.004$)$ & $0.001^{*}$ & $.096(-.004$ to .061$)$ & 0.081 \\
\hline $\begin{array}{l}\text { Low muscle mass } \\
\text { (yes/no) }\end{array}$ & $.12(.05$ to .60$)$ & $0.021^{*}$ & $-0.07(-.19$ to .44$)$ & 0.224 & $.05(-3.9$ to 9.93$)$ & 0.387 \\
\hline
\end{tabular}

* $P$ value $<0.05+\mathrm{CRP}=\mathrm{C}$-reactive protein (measure of inflammation) 
Table 2 Baseline \& follow up biochemical nutritional and inflammatory markers for patients diagnosed with low muscle mass compared with those with normal muscle mass

\begin{tabular}{|c|c|c|c|c|c|c|}
\hline \multirow[t]{2}{*}{ Nutritional variable } & \multicolumn{2}{|l|}{ Baseline mean (SD) } & \multicolumn{2}{|l|}{6 weeks } & \multicolumn{2}{|l|}{6 months } \\
\hline & $\begin{array}{l}\text { Low muscle mass } \\
(n=42)\end{array}$ & $\begin{array}{l}\text { Normal muscle } \\
\text { mass } \\
(n=360)\end{array}$ & $\begin{array}{l}\text { Low muscle mass } \\
(n=15)\end{array}$ & $\begin{array}{l}\text { Normal muscle mass } \\
(n=154)\end{array}$ & $\begin{array}{l}\text { Low muscle mass } \\
(n=9)\end{array}$ & $\begin{array}{l}\text { Normal muscle mass } \\
(n=151)\end{array}$ \\
\hline $\begin{array}{l}\text { C-reactive protein } \\
(\mathrm{mg} / \mathrm{L})\end{array}$ & $51(59)$ & $52(74)$ & $7(6)$ & $12(26)$ & $13(15)$ & $11(20)$ \\
\hline Albumin $(\mathrm{g} / \mathrm{L})+$ & $35.5(5)$ & $38.1(5) *$ & $39.6(4)$ & $40.8(3)$ & $40(5)$ & $41(3)$ \\
\hline $\begin{array}{l}\text { Red cell folate } \\
\text { (nmol/L) }\end{array}$ & $144(48)$ & $195(108) *$ & $160(45)$ & $227(117) *$ & $187(9)$ & $215(155)$ \\
\hline Riboflavin, EGRAC+ & $1.279(0.13)$ & $1.310(0.18)$ & $1.278(.15)$ & $1.273(.2)$ & $1.250(.2)$ & $1.276(.14)$ \\
\hline $\begin{array}{l}\text { Vitamin } B_{12} \text { (pmol/ } \\
\text { L) }\end{array}$ & $377(216)$ & $390(317)$ & $506(500)$ & $361(256)$ & $444(246)$ & $340(239)$ \\
\hline $\begin{array}{l}\text { Ascorbic acid } \\
(\mu \mathrm{mol} / \mathrm{L})\end{array}$ & $17(20)$ & $24(21)$ & $18(13)$ & $35(23) *$ & $47(56)$ & $30(26)$ \\
\hline $\begin{array}{l}\text { a-tocopherol } \\
(\mu \mathrm{mol} / \mathrm{L})\end{array}$ & $25(6)$ & $25(7)$ & $29(6)$ & $27(7)$ & $29(7)$ & $26(8)$ \\
\hline Carotene (umol/L) & $0.275(.3)$ & $.257(.2)$ & $.230(.08)$ & $.279(.19)$ & $272(.17)$ & $.262(.15)$ \\
\hline Retinol (umol/L) & $1.16(.5)$ & $1.22(.5)$ & $1.69(.5)$ & $1.36(.4) *$ & $1.41(.7)$ & $1.36(.5)$ \\
\hline Lycopene (umol/L) & $.121(.09)$ & $.158(.1)$ & $.108(.06)$ & $.154(.1) *$ & $.119(.1)$ & $.151(.1)$ \\
\hline Zinc (ng/ml) & $571(127)$ & $629(148) *$ & $605(110)$ & $684(150) *$ & $665(150)$ & $644(138)$ \\
\hline Copper (ng/ml) & $1110(190)$ & $1062(230)$ & $1120(241)$ & $1045(246)$ & 1036 (157) & $956(211)$ \\
\hline Selenium (ng/ml) & $63(18)$ & $68(22)$ & $68(28)$ & $83(28)$ & 84 (30) & 74 (20) \\
\hline
\end{tabular}

${ }^{*}+p$ value $<0.05$ for cumulative between $(+)$ and within group $\left(^{*}\right)$ differences at baseline or at 6 weeks, + EGRAC $=$ Erythrocyte Glutathione Reductase Activity Coefficient (EGRAC $>1.3$ indicates riboflavin biochemical deficiency)

patients with low muscle mass to this poor outcome is also not known.

Possible candidates for underlying pathophysiological mechanisms linking changes in muscle mass and mental health include malnutrition, oxidative stress and inflammation [6]., First, there is direct and indirect evidence of the role of inflammation on loss of muscle mass in older people. In older patients inflammation associated with acute and chronic illness is known to lead to decreased food intake, increased nutrients demand and loss of muscle mass [14]. Inflammation induced cytokines and reactive oxygen species can directly mediate muscle damage resulting in accumulation of oxidative damage markers such protein carbonyls known to be independently associated with low grip strength $[15,16]$. Several nutrients including those with antioxidant properties known to protect against inflammation were reported to be associated with muscle health [15-17]. Our results reveal low concentrations of a number of nutrients with antioxidant and anti-inflammatory properties in patients with low muscle mass. The ageing muscle for example, has been reported to show increased oxidative damage

Table 3 Multiple regression result of blood-borne nutritional markers on AMT, GDS and SF-36 at admission

\begin{tabular}{|c|c|c|c|c|c|c|}
\hline & Abbreviated mental test score & & Geriatric Depression Score (GDS) & & Quality of life Scores (SF-36) & \\
\hline & $\begin{array}{l}\text { Standardized Regression } \\
\text { coefficient }(95 \% \text { C.I) }\end{array}$ & $P$ value & $\begin{array}{l}\text { Standardized Regression } \\
\text { coefficient ( } 95 \% \text { C.I) }\end{array}$ & $P$ value & $\begin{array}{l}\text { Standardized Regression } \\
\text { coefficient ( } 95 \% \text { C.I) }\end{array}$ & $P$ value \\
\hline Albumin $(\mathrm{g} / \mathrm{L})+$ & $.01(-.04$ to .05$)$ & 0.780 & $-.02(-.28$ to .05$)$ & 0.154 & $1.5(.48$ to 2.5$)$ & $0.005^{*}$ \\
\hline $\begin{array}{l}\text { Red cell folate } \\
\text { (nmol/L) }\end{array}$ & $.001(-.01$ to .01$)$ & 0.527 & $-.001(-.005$ to .002$)$ & 0.395 & $-.003(-.02$ to .02$)$ & 0.756 \\
\hline $\begin{array}{l}\text { Ascorbic acid } \\
(\mu \mathrm{mol} / \mathrm{L})\end{array}$ & $.01(-.006$ to .017$)$ & 0.376 & $-.014(-.06$ to .03$)$ & 0.536 & $-.01(-.29$ to .27$)$ & 0.940 \\
\hline Retinol (umol/L) & $-.09(-.39$ to .22$)$ & 0.580 & $.24(-.89$ to 1.4$)$ & 0.674 & $-5.1(-11.9$ to 1.9$)$ & 0.151 \\
\hline $\begin{array}{l}\text { Lycopene (umol/ } \\
\text { L) }\end{array}$ & $.24(-.94$ to 1.42$)$ & 0.691 & $-3.9(-8.5$ to .75$)$ & 0.100 & $25(-.81$ to 51$)$ & 0.057 \\
\hline Zinc (ng/ml) & .001 (-.001 to .002$)$ & 0.724 & $-0.01(-.19$ to .004$)$ & 0.766 & $-.01(-.04$ to .02$)$ & 0.633 \\
\hline
\end{tabular}


Table 4 Multiple regression result of blood-borne nutritional markers on AMT, GDS and SF-36 at 6 weeks

\begin{tabular}{|c|c|c|c|c|c|c|}
\hline & \multirow{2}{*}{$\begin{array}{l}\text { Abbreviated mental test score } \\
\text { (AMT) } \\
\text { Standardized Regression } \\
\text { Coefficient ( } 95 \% \text { C.I) }\end{array}$} & \multirow[b]{2}{*}{$P$ value } & \multirow{2}{*}{$\begin{array}{l}\text { Geriatric Depression Score (GDS) } \\
\text { Standardized Regression } \\
\text { coefficient ( } 95 \% \text { C.I) }\end{array}$} & \multirow[b]{2}{*}{$P$ value } & \multirow{2}{*}{$\begin{array}{l}\text { Quality of life Scores (SF-36) } \\
\text { Standardized Regression } \\
\text { coefficient (95\%C.I) }\end{array}$} & \multirow[b]{2}{*}{$P$ value } \\
\hline & & & & & & \\
\hline Albumin $(\mathrm{g} / \mathrm{L})+$ & .06 (-.08 to .12) & 0.723 & $-.4(-.87$ to -.09$)$ & $0.017^{*}$ & $.29(-.59$ to 5.7$)$ & 0.109 \\
\hline $\begin{array}{l}\text { Red cell folate } \\
(\mathrm{nmol} / \mathrm{L})\end{array}$ & -.27 (-.003 to .001$)$ & 0.065 & .05 (-.01 to .008$)$ & 0.760 & $-.02(-.06$ to .05$)$ & 0.891 \\
\hline $\begin{array}{l}\text { Ascorbic acid } \\
(\mu \mathrm{mol} / \mathrm{L})\end{array}$ & .05 (-.001 to .02$)$ & 0.743 & $-.02(-.07$ to .06$)$ & 0.866 & $-.06(-.59$ to .40$)$ & 0.706 \\
\hline Retinol (umol/L) & .09 ( -.43 to .89$)$ & 0.490 & .04 (-2.2 to 2.8$)$ & 0.805 & -.09 ( -25 to 13$)$ & 0.565 \\
\hline $\begin{array}{l}\text { Lycopene (umol/ } \\
\text { L) }\end{array}$ & .09 (-1.9 to 3.7) & 0.515 & $.08(-7.6$ to 13.4$)$ & 0.582 & .08 (-60 to 101) & 0.610 \\
\hline Zinc (ng/ml) & $.15(-.002$ to .005$)$ & 0.396 & $.21(-.01$ to .02$)$ & 0.211 & $-.23(-.17$ to .04$)$ & 0.209 \\
\hline
\end{tabular}

* $P$ value $<0.05$

to DNA, protein and lipids. Antioxidant carotenoids quench free radicals and associated inflammation and therefore low concentrations have been found to be associated poor skeletal muscle function and disability [17]. Furthermore vitamin $\mathrm{C}$ a powerful antioxidant has also been shown to be significantly related to muscle strength in older women [18]. Treatment of old rats with a diet rich in some antioxidants and trace elements such was vitamins $\mathrm{E}$ and $\mathrm{A}$, selenium, zinc and rutin has been reported to improve the stimulation of protein synthesis in muscles by the amino acid leucine. This effect may be mediated through reduction of inflammation and associated oxidative stress however the exact mechanism is yet to be determined [19].

Although many studies have reported a relationship between low muscle mass and physical outcome many of these studies have methodological concerns and few have addressed its relationship with mental health. For example, a cross-sectional community study of 3025 older women reported no significant association between low muscle mass and cognitive impairment after adjusting for confounders. However, the analyses suggest more severe stages of sarcopenia (low mass and/or function) could be associated with cognitive impairment [20]. Another small cross-sectional study reported significant associations between depressive symptoms, cognitive impairment and sarcopenia among healthy older men living in the community [21]. A community study from Belgium of older people aged 65 years or over reported an association between sarcopenia, cognitive impairment and quality of life. The authors however acknowledged a number of study limitations including the cross-sectional design, low power and the non-representativeness of the sample [22]. A relationship between poor self-reported general health and physical functioning was also reported in a very small community sample of subjects from England diagnosed with sarcopenia, compared with those without sarcopenia [23]. The sensitivity of generic scales used by most of the above studies to detect small effects of sacropenia on quality of life has questioned [24]. Work is ongoing to test the validity of a newly developed sarcopenia-specific quality of life questionnaire [24].

Physical inactivity in hospitalised older patients during both acute illness and recovery period is another possible reason linking muscle mass loss to poor mental health [5]. Exercise and skeletal muscles signalling has been linked to brain neurogenesis and cognitive functions however the extent to which this relationship is responsible for the beneficial effects of physical activity on mental well-being is not clear [5]. Physical activity confers benefit on most risk factors of ageing, but may also improve nutritional status by increasing energy expenditure, leading to increased nutrient intake if a mixed diet is consumed [25].

Some important limitations of this study include the number of exclusions and small numbers of patents with low muscle mass at follow up visits. Another important limitation is error known to be associated with measurement of anthropometric and biochemical nutritional indices in hospitalised older people. The longitudinal design and training and assessing observer's error on anthropometric measurements, of the study and the use of a number of analyses to adjust for poor prognostic clinical indicators was to overcome some of these weaknesses.

The lack of direct correlations between blood borne micronutrients and mental health measures may partly be explained by small numbers. Nevertheless new evidence is emerging of a link between malnutrition and poor mental health and quality of life in older people and that improvement in nutritional status may leads to improvement in mental health and well-being [26]. There is an urgent need for more research in this field because the prospect of the effects of improved nutritional status of older people on mental health and quality of life could have an important and a substantial health and economic benefits. 


\section{Conclusions}

Low muscle mass in older patients is associated with poor nutritional status and mental health and wellbeing. Hitherto the evidence for the effectiveness of specific treatment or nutrient supplement is incomplete but sufficient to justify further research. Based on currently available evidence increased physical activity particularly following acute illness and a healthy diet with protein, fruits and vegetables to mitigate inflammatory responses is clearly appropriate for healthy independent ageing particularly for older patients.

\section{Abbreviations}

AMT: Abbreviated mental test questionnaire; EWGSOP: The European Working Group on Sarcopenia in Older people; GDS: Geriatric Depression questionnaire; SF-36: Medical Outcomes Study 36-items

\section{Acknowledgements}

The index study was funded by THE HEALTH FOUNDATION project grant Thank you to Dr. Sarah Forster for her help with data collection. Some of this material was presented in abstract form in a recent scientific meeting.

\section{Authors' contributions}

SG is the principal investigator, wrote the first draft, contributed to the design of the study and performed the statistical analysis and writing of the final manuscript. AA helped with data entry and analysis and drafting of the manuscript. Both authors reviewed and approved the final manuscript."

\section{Funding}

The study was supported by a research charity grant-The PPP Health Foundation, London (577/484).

\section{Availability of data and materials}

Data is available upon request to the corresponding author.

\section{Ethics approval and consent to participate}

Barnsley, UK research ethics committee approved the study by (REF: 04/ Q2304/50). Written consent was obtained from all patients recruited to this study.

\section{Consent for publication}

There is no identifiable information of the study participants in the present manuscript, therefore it is not applicable.

\section{Competing interests}

The authors declare that they have no competing interests.

Received: 26 August 2019 Accepted: 19 December 2019

Published online: 06 March 2020

\section{References}

1. Sayer AA. Low muscle mass: a research agenda has been set, but recognition in clinical practice is lagging behind. BMJ. 2010;341:952

2. Cruz-Jentoft AJ, Baeyens JP, Bauer JM, Boirie Y, Cederholm T, Landi F, et al European working group on low muscle mass in older people low muscle mass: European consensus on definition and diagnosis: report of the European working group on low muscle mass in older people. Age Ageing. 2010;39:412-23.

3. Landi F, Liperoti R, Russo A, Giovannini S, Tosato M, Capoluongo E, Bernabei R, Grazia, Onder G. Low muscle mass as a risk factor for falls in elderly individuals: Results from the iISIRENTE study. Clin Nutr. 2012. https://doi.org/ 10.1016/j.clnu.2012.02.007.

4. Rizzoli R, Reginster J, Arnal J, Bautmans I, Beaudart C, Bischoff-Ferrari H, et al. Quality of life in low muscle mass and frailty. Calcif Tissue Int. 2013;93(2): 101-20.

5. Giudice J, Taylor J. Muscle as a paracrine and endocrine organ. Curr Opin Pharmacol. 2017;34:49-55.
6. Ershler WB. A gripping reality: oxidative stress, inflammation, and the pathway to frailty. J Appl Physiol. 2007;103(1):3-5.

7. Ishii H, Makizako H, Doi T, Tsutsumimoto K, Shamada H. Associations of skeletal muscle mass, lower extremity functioning and cognitive impairment in community-dwelling older people in Japan. J Nutr Health Aging. 2019; 23(1):35-41.

8. Gariballa S, Alessa A. Association between muscle function, cognitive state, depression symptoms and quality of life of older people: evidence from clinical practice. Aging Clin Exp Res. 2018;30(4):351-7.

9. Gariballa S, Alessa A. Low muscle mass: prevalence and prognostic significance in hospitalized patients. Clin Nutr. 2013;32(5):772-6.

10. Gariballa S, Alessa A. Association between nutritional blood-based biomarkers and clinical outcome in sarcopenia patients. Clin Nutr ESPEN. 2018;25:145-8.

11. The Royal College of Physicians of London and The British Geriatrics Society. Standardised assessment scales for older people. A report of joint workshop; 1992. p. 1-27.

12. Brazier JE, Harper R, Jones NMB, O'Cathain A, Thomas KJ, Usherwood T, Westlake L. Validating the SF-36 health survey questionnaire: new outcome measure for primary care. BMJ. 1992;305:160-4.

13. Burns JM, Johnson DK, Watts A, et al. Reduced lean mass in early Alzheimer disease and its association with brain atrophy. Arch Neurol. 2010;67:428-33.

14. Gariballa SE. Malnutrition in hospitalised elderly patients: when does it matter? Clin Nutr. 2001;20(6):487-91.

15. Fulle S, Protasi F, Di Tano G, et al. The contribution of reactive oxygen species to sarcopenia and muscle ageing. Exp Gerontol. 2004;39:17-24.

16. Howard C, Ferrucci $L$, Sun $K$, et al. Oxidative protein damage is associated with poor grip strength among older women living in the community. J Appl Physiol. 2007;103:17-20.

17. Semba RD, Lauretani F, Ferruci L. Carotenoids as protection against sarcopenia in older adults. Arch Biochem Biophys. 2007:458:141-5.

18. Saito $\mathrm{K}$, Yokoyama $\mathrm{T}$, Yoshida $\mathrm{H}$, et al. Concentration of plasma vitamin $\mathrm{C}$ in community-dwelling elderly Japanese women significant determinant of muscle strength and physical performance. J Gerontol A Biol Sci Med Sci doi. 2011. https://doi.org/10.1093/gerona/glr174.

19. Marzani B, Balage M, Venien A, et al. Antioxidant supplementation restores defective leucine stimulation of protein synthesis in skeletal muscle from old rats. J Nutr. 2008;138:2205-11.

20. Abellan van Kan G, Cesari M, Gillette-Guyonnet S, Dupuy C, Nourhashémi F, Schott AM, Beauchet O, Annweiler C, Vellas B, Rolland Y. Low muscle mass and cognitive impairment in elderly women: results from the EPIDOS cohort. Age Ageing. 2013;42(2):196-202.

21. Hsu YH, Liang CK, Chou MY, Liao MC, Lin YT, Chen LK, Lo YK. Association of cognitive impairment, depressive symptoms and Low muscle mass among healthy older men in the veterans retirement community in southern Taiwan: a cross-sectional study. Geriatr Gerontol Int. 2014;14(Suppl 1):102-8.

22. Beaudart C, Reginster JY, Petermans J, Gillain S, Quabron A, Locquet M, Slomian J, Buckinx F, Bruyère O. Quality of life and physical components linked to Low muscle mass: The SarcoPhAge study. Exp Gerontol. 2015;69:103-10.

23. Patel HP, Syddall HE, Jameson K, Robinson S, Denison H, Roberts HC, Edwards M, Dennison E, Cooper C, Aihie SA. Prevalence of Low muscle mass in community-dwelling older people in the UK using the European Working Group on Low muscle mass in Older People (EWGSOP) definition: findings from the Hertfordshire Cohort Study (HCS). Age Ageing. 2013;42(3):378-84

24. Beaudart C, Biver E, Reginster J, Rizzoli R, Rolland Y, Bautmans I, et al. Development of a self-administrated quality of life questionnaire for sarcopenia in elderly subjects: the SarQoL. Age Ageing. 2015;44(6):960-6.

25. Wiseman M, Jackson A. Nutrition in health and disease. Clin Med. 2004;4: 397-400.

26. Gariballa SE. International Handbook of Behavior, Diet and Nutrition. In: Preedy VR, et al., editors. Handbook of Behavior, Food and Nutrition; 2011. p. 3109-311.

\section{Publisher's Note}

Springer Nature remains neutral with regard to jurisdictional claims in published maps and institutional affiliations. 